\title{
A NOTE ON DIFFERENTIABLE FUNCTIONS
}

\author{
RICHARD J. FLEISSNER AND JAMES FORAN
}

\begin{abstract}
This paper provides a proof that the class of those real functions $f$ for which there exists a change of variable $g$ so that $f \circ g$ is differentiable coincides with the class of continuous functions which are of generalized bounded variation in the restricted sense.
\end{abstract}

The purpose of this note is to furnish the proof of a theorem stated in [1] which had been communicated privately to its authors. The definitions of $V B G_{*}, A C G_{*}$, Lusin's condition $(\mathrm{N})$ and Banach's condition $\left(\mathrm{T}_{1}\right)$ may be found in [2].

THEOREM. A function $F$ can be transformed by means of an inner homeomorphism into an everywhere differentiable function if and only if $F$ is continuous and of generalized bounded variation in the restricted sense $\left(V B G_{*}\right)$.

Proof. As noted in [1], the necessity of continuity and $V B G_{*}$ is obvious since these conditions are preserved under inner homeomorphisms and are satisfied by every differentiable function [2, p. 234].

Let $F$ be a continuous, $V B G_{*}$ function. Then $F$ satisfies Banach's condition $\left(\mathrm{T}_{1}\right)$ [2, p. 279]. Consequently, $F=G \circ H$, where $G$ is absolutely continuous and $H$ is of bounded variation [2, p. 287]. Bruckner and Goffman [1] have shown that there exists a homeomorphism $U$ such that $H \circ U$ is differentiable. Since differentiable functions satisfy condition $(\mathrm{N})[2$, Theorem $6.5, \mathrm{p}$. 227] and since $G$ is absolutely continuous, $F \circ U=G \circ(H \circ U)$ satisfies condition (N). As noted in the proof of necessity, $F \circ U$ is continuous and $V B G_{*}$. Thus $F \circ U$ is $A C G_{*}$ [2, Theorem 6.7, p. 227 and Theorem 8.8, p. 233]. Finally, Tolstov [3] has shown that each $A C G_{*}$ function can be transformed into a differentiable function by an inner homeomorphism.

\section{REFERENCES}

1. A. M. Bruckner and C. Goffman, Differentiability through change of variables, Proc. Amer. Math. Soc. 61 (1976), 235-241.

2. S. Saks, Theory of the integral, Dover, New York, 1937.

3. G. Tolstov, Parametric differentiation and the narrow sense Denjoy integral, Mat. Sb. 53 (95) (1961), 387-392. (Russian)

Department of Mathematics, Western Illinois University, Macomb, Illinois 61455

Department of Mathematics, University of Missouri, Kansas City, Missouri 64110

Received by the editors July 29, 1977.

AMS (MOS) subject classifications (1970). Primary 26A45. 